\title{
Multilatinas and International Business Studies
}

\author{
Alvaro Cuervo-Cazurra, Northeastern University, USA
}

\section{Introduction}

Multilatinas, i.e., multinational firms originating in Latin America, are increasingly playing a dominant role in global business. In the last three decades, many evolved from exporters to become regional leaders in the Americas, like Mexican telecommunications firm América Movil or Argentinean candy producer Arcor. Some have even become global leaders, like Brazilian airplane manufacturer Embraer, Argentinean steel tube maker Techint, or the Mexican cement producer Cemex. However, many multilatinas are little known outside their region, or even within it. One reason is their strategies. Many serve other companies rather than final consumers, and those operating in consumer goods tend to hide their country of origin and use foreign brands in their international expansion. Another reason is limited attention among researchers. ${ }^{1}$ Much of the interest in multinationals from emerging markets has concentrated on firms from the so-called BRIC countries (Brazil, Russia, India, China) even though the vast differences among these nations hamper meaningful comparisons.

This lack of knowledge on multilatina strategies is a missed opportunity. It is not just because they are an exciting and increasingly important phenomenon. It is because multilatinas can help us understand the influence of the home country on firms' internationalization in ways that cannot be achieved by examining firms elsewhere. Latin American countries share deep cultural, economic, and political historical commonalities, unlike nations in other emerging regions, such as Africa, Asia, or Eastern Europe. These commonalities enable academics to understand better which and how country characteristics affect firms' internationalization. ${ }^{2}$ Such knowledge is also important for managers because lessons derived from the study of the internationalization of multilatinas in one country might be highly valuable in others given the similarities in home country conditions; insights from firms in other regions may be of little applicability given the large differences among home countries.

\section{Commonalities across Latin America}

The historical evolution of Latin American countries has resulted in many commonalities that shaped firm strategies. The colonial period, starting in the $15^{\text {th }}$ century, led to considerable similarities in socio-cultural development. The colonial powers (Spain, Portugal, and France) imposed a hierarchical social structure with an embedded preference for European descendants; a civil law legal tradition; a bureaucratic and centralized state structure; Romance languages that have mostly superseded indigenous ones; Catholicism; and a system of natural resource extraction that exploited natives and imported slaves, among other factors. Firms were unsophisticated, dedicated to exporting commodities to their colonial power and dependent on the import of sophisticated products from it.

The post-independence period, beginning in the early $19^{\text {th }}$ century, reinforced commonalities among countries and resulted in inward-looking firms. Politically, newly independent countries followed a cycle of democratic governments, which mostly represented the wealthy, followed by military dictatorships, and then return to civilian rule. This cycle reinforced weak institutions and exclusion of the poor and non-whites, leading to internal conflicts and violent military rulers in the $20^{\text {th }}$ century. Economically, Latin America developed by exploiting commodities and open markets. However, after the first third of the $20^{\text {th }}$ century, governments generally adopted an import substitution economic model, imposing barriers against foreign goods and firms, taking an active role in industrialization, 
creating state-owned firms, and providing protected markets for local producers. This resulted in companies that were not internationally competitive and focused on serving the domestic market. Firms with government connections enjoyed financial and regulatory support that led to industry diversification and the emergence of business groups.

The last third of the $20^{\text {th }}$ century witnessed a deep economic and political opening that led to the internationalization of firms. Import substitution was replaced with pro-market reforms, starting in the 1970s and deepening in the 1990s. The oil shocks of the 1970s and unsustainable government borrowing created a deep economic crisis in the 1980s, known as the "lost decade," that led to hyperinflation and sharp rises in poverty. To address this, in the 1980s and 1990s governments implemented profound economic transformations by following the so-called Washington Consensus program of pro-market reforms. Governments privatized state-owned firms, deregulated industries and international trade and investment, and liberalized prices. In parallel, in the 1980s Latin American countries underwent a democratic transformation. Military rulers were ousted as they proved inept at managing the economic crisis, while the US withdrew support for dictatorships and promoted democracy as the Cold War ended. As a result, the 1990s became a golden decade. Latin American countries experienced substantial and sustained economic growth and reductions in poverty through trickle-down economics and active social redistribution programs. Companies that managed to adapt and survive the economic transformation, and newly created firms, were much more competitive. Their exposure to imports and foreign competitors forced them to upgrade their capabilities, which enabled some of them to reach international levels of competitiveness and expand abroad.

By the $21^{\text {st }}$ century, Latin American economies had reached middle-income status, and their firms were increasingly globalized. In the 2000s, doubts about the success of pro-market reforms and some short-lived crises led some newly-elected leftist governments to increase economic controls. However, by the late 2010s, most Latin American countries had not only entrenched democracies but also demanding citizens that supported the active prosecution of corruption among the political and business elite, stable economies with sensible macroeconomic policies, vanishing armed conflicts, and growing middle classes. Firms improved their capabilities and started processes of indigenous innovation. Many saw international markets as a viable avenue for additional growth and scale, and some ventured not only into neighboring countries but farther, achieving global player status.

Statistics echo these historical commonalities among Latin American countries and sharp differences among other economies. Tables 1 and 2 provide indicators of the social and economic conditions of Latin American countries, the other BRICS emerging countries, and the leading advanced economies.
There are significant socio-cultural resemblances among Latin American countries, such as common language, racial distribution, dominant religion, and urbanization. Countries show economic similitudes such as relatively high average income for emerging economies, openness to international trade, and intra-region main trading partners. Outward foreign direct investment tends to concentrate in the region, but much of the inward foreign direct investment comes from advanced countries with historical ties, USA and Spain, as well as neighboring countries.

In contrast, countries in other regions show vast differences. The traditionally analyzed BRICS countries have little in common, other than their selection by the consulting firm BCG as countries of the future. They differ in socio-cultural structure, as well as political and economic systems, and their international trade and investment partners show remarkable diversity, except for one unusual commonality: probable round-tripping of foreign direct investment, given that offshore financial centers (Hong Kong and the British Virgin Islands for China, Mauritius for India, and Cyprus and the Netherlands for Russia) are both the source and destination of much foreign direct investment. Advanced economies also have limited similarities, other than high development levels and stable democracies; they are different in socio-cultural characteristics, legal and colonial history; and show a remarkable variety in their international trade and investment patterns. A similar conclusion can be arrived at when studying characteristics of firms in other emerging regions, like Africa, Asia, or Eastern Europe.

\section{Multilatinas and International Busi= ness Models}

This comparison highlights how historical commonalities among Latin American countries can help understand better the influence of home country conditions on firm internationalization. The similarities act as an implicit control of country characteristics that cannot be achieved in other regions. I now outline four suggestions on how to do this.

First, geographically, multilatinas are a natural laboratory for studying how the geographic distance to a sizeable advanced market affects country selection in internationalization. The traditional incremental internationalization process model proposes that firms will expand to nearby countries first and then to more distant countries later. However, multilatinas can select between expanding into nearby countries that are very similar to their home country but offer fewer market opportunities, or expanding into the US that is different from their home market but a much larger potential market. Distance to the US, ranging from bordering Mexico to far away Chile and Argentina, can help discern better the role of geographic distance in international trade and investment decisions. 
Table 1. Comparison of demographic characteristics of Latin American and selected countries

\begin{tabular}{|c|c|c|c|c|c|c|c|}
\hline Country & $\begin{array}{l}\text { Population, } \\
\text { mn }\end{array}$ & Ethnic groups (\% total), top 3 & $\begin{array}{l}\text { Main language } \\
\text { ( } \% \text { spoken at } \\
\text { home) }\end{array}$ & $\begin{array}{l}\text { Religion ( } \% \text { total), top } 3 \text { above } \\
5 \%\end{array}$ & $\begin{array}{c}\text { Urbanization, } \\
\%\end{array}$ & $\begin{array}{l}\text { Life expec- } \\
\text { tancy, years }\end{array}$ & $\begin{array}{l}\text { Literacy, } \% \\
\text { over } 15 \text { yrs } \\
\text { old }\end{array}$ \\
\hline \multicolumn{8}{|c|}{ Latin America } \\
\hline Argentina & 45 & White/Mestizo (97), Amerindian (2), Black (0.4) & Spanish & Catholic (92) & 91 & 78 & 99 \\
\hline Bolivia & 11 & Mestizo (68), Amerindian (20), White (5) & Spanish (61) & $\begin{array}{l}\text { Catholic (77), Evangelical (8), } \\
\text { Protestant (8) }\end{array}$ & 69 & 70 & 93 \\
\hline Brazil & 209 & White (48), Mulatto (43), Black (8) & Portuguese & Catholic (70), Protestant (22) & 87 & 74 & 92 \\
\hline Chile & 18 & White/Mestizo (89), Amerindian (11) & Spanish (100) & Catholic (67), Protestant (16) & 88 & 79 & 97 \\
\hline Colombia & 48 & $\begin{array}{l}\text { Mestizo/White (84), African/Mulatto (10), } \\
\text { Amerindian (3) }\end{array}$ & Spanish & Catholic (79), Protestant (14) & 81 & 76 & 94 \\
\hline Costa Rica & 5 & White/Mestizo (84), Mulatto (7), Amerindian (2) & Spanish & $\begin{array}{l}\text { Catholic (72), Evangelical/ } \\
\text { Pentecostal (12) }\end{array}$ & 79 & 79 & 98 \\
\hline Cuba & 11 & White (64), Mulatto/Mixed (27), Black (9) & Spanish & Christian (59), Folk (17) & 77 & 79 & 100 \\
\hline $\begin{array}{l}\text { Dominican } \\
\text { Republic }\end{array}$ & 10 & Mixed (70), Black (16), White (14) & Spanish & Catholic (95) & 81 & 71 & 94 \\
\hline Ecuador & 16 & Mestizo (79), Amerindian (7), White (6) & Spanish (93) & Catholic (74), Evangelical (10) & 64 & 77 & 94 \\
\hline El Salvador & 6 & Mestizo (86), White (13), Amerindian (0.2) & Spanish & Catholic (50), Protestant (36) & 72 & 75 & 88 \\
\hline Guatemala & 17 & Mestizo/White (60), Amerindian (39) & Spanish (69) & Catholic, Protestant, Indigenous & 51 & 72 & 82 \\
\hline Haiti & 11 & Black (95), Mixed/White (5) & French \& Creole & Catholic (55), Protestant (29) & 95 & 78 & 99 \\
\hline Honduras & 9 & Mestizo (90), Amerindian (7), Black (2) & $\begin{array}{l}\text { Spanish \& } \\
\text { Amerindian }\end{array}$ & Catholic (46), Protestant (41) & 57 & 71 & 89 \\
\hline Mexico & 126 & Mestizo (62), Amerindian (28), White (10) & Spanish (93) & Catholic (83), Evangelical (5) & 80 & 76 & 95 \\
\hline Nicaragua & 6 & Mestizo (69), White (17), Black (9) & Spanish (95) & Catholic (50), Evangelical (33) & 59 & 74 & 83 \\
\hline Panama & 4 & Mestizo (65), Amerindian (12.3), Black (9) & Spanish & Catholic (85), Protestant (15) & 68 & 79 & 95 \\
\hline Paraguay & 7 & Mestizo (95) & $\begin{array}{l}\text { Spanish \& } \\
\text { Guarani (46) }\end{array}$ & Catholic (90), Protestant (6) & 62 & 78 & 95 \\
\hline Peru & 31 & Mestizo (60) Amerindian (26), White (6) & Spanish (83) & Catholic (60), Evangelical (11) & 78 & 74 & 94 \\
\hline Uruguay & 3 & White (88), Black (5), Amerindian (2) & Spanish & $\begin{array}{l}\text { Catholic (47), Non-denomi- } \\
\text { national (23), Non-Catholic } \\
\text { Christian (11) }\end{array}$ & 95 & 78 & 99 \\
\hline Venezuela & 32 & n.a. & Spanish & Catholic (96) & 88 & 76 & 97 \\
\hline \multicolumn{8}{|c|}{ Emerging economies } \\
\hline China & 1385 & Han Chinese (92), Zhuang (1) & Chinese & $\begin{array}{l}\text { Folk (22), Buddhist (18), Chris- } \\
\text { tian (5) }\end{array}$ & 59 & 76 & 96 \\
\hline India & 1297 & Indo-Aryan (72), Dravidian (25), Mongoloid (3) & Hindi (44) & Hindu (80), Muslim (14) & 34 & 69 & 71 \\
\hline Russia & 142 & Russian (78), Tatar (4), Ukrainian (1) & Russian (86) & $\begin{array}{l}\text { Russian Orthodox (17), Muslim } \\
\text { (13) }\end{array}$ & 74 & 66 & 99 \\
\hline South Africa & 55 & Black (81), Colored (9), White (8) & IsiZulu (25) & Christian (86), Traditional (5) & 66 & 64 & 94 \\
\hline \multicolumn{8}{|c|}{ Advanced economies } \\
\hline France & 67 & n.a. & French (100) & Christian (65), Muslim (8) & 80 & 82 & 100 \\
\hline Germany & 81 & German (87), Turkish (2), Polish (1) & German & $\begin{array}{l}\text { Catholic (28), Protestant (26), } \\
\text { Muslim (5) }\end{array}$ & 77 & 81 & 100 \\
\hline Japan & 126 & Japanese (98), Chinese (0.5), Korean (0.4) & Japanese & Shinto (70), Buddhist (70) & 92 & 86 & 100 \\
\hline $\begin{array}{l}\text { United } \\
\text { Kingdom }\end{array}$ & 65 & White (87), Black (3), Asian (2) & English & Christian (60) & 83 & 81 & 100 \\
\hline United States & 329 & White (72), Black (13), Asian (5) & English & Protestant (47), Catholic (21) & 82 & 80 & 100 \\
\hline
\end{tabular}

Source: Created using information from CIA (2019). Figures rounded from the original.

Note: n.a. not applicable/available. Latin American countries are independent countries that were former Spanish, Portuguese, and French colonies. 
Table 2. Comparison of economic characteristics of Latin American and selected countries

\begin{tabular}{|c|c|c|c|c|c|c|c|c|c|c|c|}
\hline Country & $\begin{array}{l}\text { GDP, } \\
\text { PPP } \\
\text { US\$ } \\
\text { bn }\end{array}$ & $\begin{array}{l}\text { GDP, } \\
\text { US\$ } \\
\text { bn }\end{array}$ & $\begin{array}{l}\text { GDP per } \\
\text { capita } \\
\text { PPP, USS } \\
\text { th }\end{array}$ & $\begin{array}{l}\text { Imports, } \\
\text { US\$ bn }\end{array}$ & $\begin{array}{l}\text { Imports (\% total), } \\
\text { top } 3\end{array}$ & $\begin{array}{l}\text { Exports, } \\
\text { US\$ bn }\end{array}$ & Exports $(\% \text { total })_{1}$, top 3 & $\begin{array}{l}\text { Inward } \\
\text { FDI } \\
\text { stock, } \\
\text { US\$bn }\end{array}$ & $\begin{array}{l}\text { Inward FDI stock (\% } \\
\text { total), top } 3\end{array}$ & $\begin{array}{l}\text { Outward } \\
\text { FDI stock, } \\
\text { USS bn }\end{array}$ & $\begin{array}{l}\text { Outward FDI stock (\% } \\
\text { total), top } 3\end{array}$ \\
\hline \multicolumn{12}{|c|}{ Latin America } \\
\hline Argentina & 922 & 674 & 21 & 64 & $\begin{array}{l}\text { Brazil (27), China } \\
\text { (19), USA (11) }\end{array}$ & 58 & $\begin{array}{l}\text { Brazil (16), USA (8), } \\
\text { China (8) }\end{array}$ & 77 & $\begin{array}{l}\text { Spain (20), USA (19) } \\
\text {, Netherlands (9) }\end{array}$ & 41 & $\begin{array}{l}\text { Uruguay (55), Chile (39), } \\
\text { Mexico (13) }\end{array}$ \\
\hline Bolivia & 84 & 38 & 8 & 9 & $\begin{array}{l}\text { China (22), Brazil } \\
\text { (17), Argentina (13) }\end{array}$ & 8 & $\begin{array}{l}\text { Brazil (18), Argentina } \\
\text { (16), USA (8) }\end{array}$ & 12 & $\begin{array}{l}\text { Spain (30), Brazil } \\
\text { (12), UK (11) }\end{array}$ & 1 & $\begin{array}{l}\text { Argentina (79), Peru (13), } \\
\text { Bangladesh (5) }\end{array}$ \\
\hline Brazil & 3248 & 2055 & 16 & 153 & $\begin{array}{l}\text { China (18), USA } \\
\text { (17), Argentina (6) }\end{array}$ & 217 & $\begin{array}{l}\text { China (22), USA (13), } \\
\text { Argentina (8) }\end{array}$ & 778 & $\begin{array}{l}\text { Netherlands (29), } \\
\text { USA (15), Spain (11) }\end{array}$ & 359 & $\begin{array}{l}\text { Austria (21), Cayman Is. } \\
\text { (16), Netherlands (11) }\end{array}$ \\
\hline Chile & 452 & 277 & 25 & 61 & $\begin{array}{l}\text { China (24), USA } \\
\text { (18), Brazil (9) }\end{array}$ & 69 & $\begin{array}{l}\text { China (28), USA (15), } \\
\text { Japan (9) }\end{array}$ & 206 & $\begin{array}{l}\text { Spain (18), USA } \\
\text { (16), Canada (9) }\end{array}$ & 124 & $\begin{array}{l}\text { Argentina (18). Brazil } \\
\text { (17), Peru (13) }\end{array}$ \\
\hline Colombia & 712 & 315 & 14 & 44 & $\begin{array}{l}\text { USA (26), China } \\
\text { (19), Mexico (8) }\end{array}$ & 9 & $\begin{array}{l}\text { USA (29), Panama (9), } \\
\text { China (5) }\end{array}$ & 180 & $\begin{array}{l}\text { Spain (31), USA } \\
\text { (24), Mexico (14) }\end{array}$ & 56 & $\begin{array}{l}\text { Chile (35), Panama (33), } \\
\text { Peru (13) }\end{array}$ \\
\hline Costa Rica & 84 & 58 & 17 & 15 & $\begin{array}{l}\text { USA (38), China } \\
\text { (13), Mexico (7) }\end{array}$ & 11 & $\begin{array}{l}\text { USA (41), Belgium (6), } \\
\text { Panama (6) }\end{array}$ & 34 & $\begin{array}{l}\text { USA (61), Spain (7), } \\
\text { Mexico (5) }\end{array}$ & 4 & $\begin{array}{l}\text { USA (61), Spain (7), } \\
\text { Mexico (5) }\end{array}$ \\
\hline Cuba & 137 & 94 & 12 & 11 & $\begin{array}{l}\text { China (22), Spain } \\
\text { (14), Russia (5) }\end{array}$ & 3 & $\begin{array}{l}\text { Venezuela (18), Spain } \\
\text { (12), Russia (8) }\end{array}$ & n.a. & n.a. & 4 & n.a. \\
\hline $\begin{array}{l}\text { Dominican } \\
\text { Republic }\end{array}$ & 173 & 76 & 17 & 18 & $\begin{array}{l}\text { USA (41), China } \\
\text { (14), Mexico (5) }\end{array}$ & 10 & $\begin{array}{l}\text { USA (51), Haiti (9), } \\
\text { Canada (8) }\end{array}$ & 37 & $\begin{array}{l}\text { Mexico (40), Spain } \\
\text { (23), USA (22) }\end{array}$ & 1 & $\begin{array}{l}\text { Panama (55), USA (20), } \\
\text { Argentina (20) }\end{array}$ \\
\hline Ecuador & 193 & 104 & 12 & 19 & $\begin{array}{l}\text { USA (23), China } \\
\text { (15), Colombia (9) }\end{array}$ & 19 & $\begin{array}{l}\text { USA (32), Vietnam (8), } \\
\text { Peru (7) }\end{array}$ & 17 & $\begin{array}{l}\text { Spain (42), Mexico } \\
\text { (23), USA (19) }\end{array}$ & 6 & $\begin{array}{l}\text { Panama (40), Peru (34), } \\
\text { USA (11) }\end{array}$ \\
\hline $\begin{array}{l}\text { El } \\
\text { Salvador }\end{array}$ & 51 & 25 & 8 & 10 & $\begin{array}{l}\text { USA (37), Guatemala } \\
\text { (11), China (9) }\end{array}$ & 5 & $\begin{array}{l}\text { USA (46), Honduras } \\
\text { (14), Guatemala (14) }\end{array}$ & 10 & $\begin{array}{l}\text { USA (32), Panama } \\
(29), \text { Mexico (10) }\end{array}$ & 1 & Nicaragua (88) \\
\hline $\begin{array}{l}\text { Guate- } \\
\text { mala }\end{array}$ & 138 & 76 & 8 & 17 & $\begin{array}{l}\text { USA (40), China } \\
\text { (11), Mexico (11) }\end{array}$ & 11 & $\begin{array}{l}\text { USA (34), El Salvador } \\
\text { (11), Honduras (9) }\end{array}$ & 16 & $\begin{array}{l}\text { USA (29), Mexico } \\
\text { (17), Colombia (9) }\end{array}$ & n.a. & $\begin{array}{l}\text { Panama (31), Bahamas } \\
\text { (31), Barbados (30) }\end{array}$ \\
\hline Haiti & 78 & 59 & 2 & 9 & $\begin{array}{l}\text { China (20), Brazil } \\
\text { (20), Argentina (13) }\end{array}$ & 11 & $\begin{array}{l}\text { China (19), Brazil (16), } \\
\text { USA (6) }\end{array}$ & 45 & $\begin{array}{l}\text { USA (80), Italy (13), } \\
\text { Korea (7) }\end{array}$ & 20 & n.a. \\
\hline Honduras & 46 & 23 & 6 & 11 & $\begin{array}{l}\text { USA (40), Guatemala } \\
\text { (11), China (9) }\end{array}$ & 9 & $\begin{array}{l}\text { USA (35), Germany (9), } \\
\text { Belgium (8) }\end{array}$ & 8 & $\begin{array}{l}\text { USA (23), Mexico } \\
\text { (16), UK (14) }\end{array}$ & 0 & $\begin{array}{l}\text { Costa Rica (43), El Salva- } \\
\text { dor (42), Colombia (11) }\end{array}$ \\
\hline Mexico & 2463 & 1151 & 20 & 421 & $\begin{array}{l}\text { USA (46), China } \\
\text { (18), Japan (4) }\end{array}$ & 410 & USA (80) & 554 & $\begin{array}{l}\text { USA (55), Spain (12), } \\
\text { Netherlands (10) }\end{array}$ & 244 & $\begin{array}{l}\text { USA (33), Brazil (17), } \\
\text { Spain (13) }\end{array}$ \\
\hline Nicaragua & 36 & 14 & 6 & 7 & $\begin{array}{l}\text { USA (21), China } \\
\text { (14), Mexico (11) }\end{array}$ & 4 & $\begin{array}{l}\text { USA (44), El Salvador } \\
\text { (6), Venezuela (6) }\end{array}$ & 1 & $\begin{array}{l}\text { Mexico (58), USA } \\
\text { (36) }\end{array}$ & 1 & $\begin{array}{l}\text { Panama (90), Mexico (7), } \\
\text { Poland (4) }\end{array}$ \\
\hline Panama & 104 & 62 & 25 & 22 & $\begin{array}{l}\text { USA (24), China } \\
\text { (10), Mexico (5) }\end{array}$ & 16 & $\begin{array}{l}\text { USA (19), Netherlands } \\
\text { (17), China (7) }\end{array}$ & 57 & $\begin{array}{l}\text { USA (18), UK (13), } \\
\text { Colombia (10) }\end{array}$ & 11 & $\begin{array}{l}\text { Chile (27), El Salvador } \\
\text { (18), Turkey (17) }\end{array}$ \\
\hline Paraguay & 89 & 39 & 13 & 11 & $\begin{array}{l}\text { China (31), Brazil } \\
\text { (23), Argentina (13) }\end{array}$ & 12 & $\begin{array}{l}\text { Brazil (32), Argentina } \\
\text { (16), Chile (7) }\end{array}$ & 6 & $\begin{array}{l}\text { USA (50), Brazil } \\
\text { (14), Argentina (8) }\end{array}$ & 1 & $\begin{array}{l}\text { Uruguay (86), Argentina } \\
\text { (17), South Africa (8) }\end{array}$ \\
\hline Peru & 430 & 214 & 14 & 39 & $\begin{array}{l}\text { China (22), USA } \\
\text { (20), Brazil (6) }\end{array}$ & 45 & $\begin{array}{l}\text { China (27), USA (15), } \\
\text { Switzerland (6) }\end{array}$ & 98 & $\begin{array}{l}\text { Spain (19), USA } \\
\text { (14), UK (20) }\end{array}$ & 5 & $\begin{array}{l}\text { Chile (30), USA (22), } \\
\text { Panama (17) }\end{array}$ \\
\hline Uruguay & 78 & 59 & 22 & 9 & $\begin{array}{l}\text { China (20), Brazil } \\
\text { (20), Argentina (12) }\end{array}$ & 11 & $\begin{array}{l}\text { China (19), Brazil (16), } \\
\text { USA (6) }\end{array}$ & 45 & $\begin{array}{l}\text { Argentina (27), Brazil } \\
\text { (8), Spain (7) }\end{array}$ & 20 & $\begin{array}{l}\text { Argentina (40), Spain } \\
(37), \text { Italy (7) }\end{array}$ \\
\hline Venezuela & 382 & 210 & 13 & 11 & $\begin{array}{l}\text { USA (25), China } \\
\text { (14), Mexico (10) }\end{array}$ & 32 & $\begin{array}{l}\text { USA (35), India (18), } \\
\text { China (16) }\end{array}$ & 33 & $\begin{array}{l}\text { Netherlands (17), } \\
\text { USA (16), France (7) }\end{array}$ & 35 & $\begin{array}{l}\text { USA (70), Spain (19), } \\
\text { Panama (8) }\end{array}$ \\
\hline \multicolumn{12}{|c|}{ Emerging economies } \\
\hline China & 23210 & 12010 & 17 & 1740 & $\begin{array}{l}\text { South Korea (10), } \\
\text { Japan (9), USA (9) }\end{array}$ & 2216 & $\begin{array}{l}\text { USA (19), Hong Kong } \\
\text { (12), Japan (6) }\end{array}$ & 1523 & $\begin{array}{l}\text { Hong Kong (44), } \\
\text { British Virgin Is. (10), } \\
\text { USA (7) }\end{array}$ & 1383 & $\begin{array}{l}\text { Hong Kong (58), British } \\
\text { Virgin Is. (6), Cayman } \\
\text { Is. (6) }\end{array}$ \\
\hline India & 9474 & 2604 & 7 & 452 & $\begin{array}{l}\text { China (16), USA (6), } \\
\text { UAE (5) }\end{array}$ & 304 & $\begin{array}{l}\text { USA (16), UAE (10), } \\
\text { Hong Kong (5) }\end{array}$ & 378 & $\begin{array}{l}\text { Mauritius (27), UK } \\
\text { (16), USA (15) }\end{array}$ & 155 & $\begin{array}{l}\text { Singapore (27), Mauritius } \\
\text { (16), Netherlands (14), }\end{array}$ \\
\hline Russia & 4016 & 1578 & 28 & 238 & $\begin{array}{l}\text { China (21), Germany } \\
\text { (11), USA (6) }\end{array}$ & 353 & $\begin{array}{l}\text { China (11), Netherlands } \\
(10), \text { Germany (7) }\end{array}$ & 535 & $\begin{array}{l}\text { Cyprus (30), Neth- } \\
\text { erlands (12), British } \\
\text { Virgin Is. (10) }\end{array}$ & 471 & $\begin{array}{l}\text { Cyprus (37), Netherlands } \\
\text { (16), British Virgin Is. (12) }\end{array}$ \\
\hline $\begin{array}{l}\text { South } \\
\text { Africa }\end{array}$ & 767 & 349 & 14 & 89 & $\begin{array}{l}\text { China (18), Germany } \\
\text { (12), USA (7) }\end{array}$ & 95 & $\begin{array}{l}\text { China (10), USA (8), } \\
\text { Germany (7) }\end{array}$ & 157 & $\begin{array}{l}\text { UK (46), Netherlands } \\
\text { (19), USA (7) }\end{array}$ & 270 & $\begin{array}{l}\text { China (18), UK (16), } \\
\text { Mauritius (10) }\end{array}$ \\
\hline
\end{tabular}




\begin{tabular}{|c|c|c|c|c|c|c|c|c|c|c|c|}
\hline \multicolumn{12}{|c|}{ Advanced economies } \\
\hline France & 2856 & 2588 & 44 & 602 & $\begin{array}{l}\text { Germany (19), } \\
\text { Belgium (109), } \\
\text { Netherlands (8) }\end{array}$ & 550 & $\begin{array}{l}\text { Germany (15), Spain } \\
\text { (8), Italy (8) }\end{array}$ & 858 & $\begin{array}{l}\text { Netherlands (17), } \\
\text { Luxembourg (13), } \\
\text { Belgium (13) }\end{array}$ & 1429 & $\begin{array}{l}\text { USA (15), Belgium (14), } \\
\text { Netherlands (12), }\end{array}$ \\
\hline Germany & 4199 & 3701 & 51 & 1135 & $\begin{array}{l}\text { Netherlands (14), } \\
\text { China (7), France (7) }\end{array}$ & 1434 & $\begin{array}{l}\text { USA (9), France (8), } \\
\text { China (7) }\end{array}$ & 1653 & $\begin{array}{l}\text { Netherlands (24), } \\
\text { Luxembourg (14), } \\
\text { USA (9) }\end{array}$ & 2298 & $\begin{array}{l}\text { USA (22), UK (10), } \\
\text { Netherland (7) }\end{array}$ \\
\hline Japan & 5443 & 4873 & 43 & 645 & $\begin{array}{l}\text { China (25), USA } \\
\text { (11), Australia (6) }\end{array}$ & 689 & $\begin{array}{l}\text { USA (20), China (19), } \\
\text { South Korea (8) }\end{array}$ & 253 & $\begin{array}{l}\text { USA (30), Nether- } \\
\text { lands (15), France (9) }\end{array}$ & 1547 & $\begin{array}{l}\text { USA (28), Netherlands } \\
\text { (9), China (9) }\end{array}$ \\
\hline $\begin{array}{l}\text { United } \\
\text { Kingdom }\end{array}$ & 2925 & 2628 & 44 & 616 & $\begin{array}{l}\text { Germany (14), USA } \\
\text { 910), China (9) }\end{array}$ & 441 & $\begin{array}{l}\text { USA (13), Germany } \\
\text { (11), France (7) }\end{array}$ & 2078 & $\begin{array}{l}\text { USA (29), Nether- } \\
\text { lands (15), France (9) }\end{array}$ & 2110 & $\begin{array}{l}\text { USA (19), Luxembourg } \\
\text { (13), Netherlands (12) }\end{array}$ \\
\hline $\begin{array}{l}\text { United } \\
\text { States }\end{array}$ & 19490 & 19490 & 60 & 2361 & $\begin{array}{l}\text { China (22), Mexico } \\
\text { (13), Canada (13) }\end{array}$ & 1553 & $\begin{array}{l}\text { Canada (18), Mexico } \\
\text { (16), China (8) }\end{array}$ & 4080 & $\begin{array}{l}\text { UK (18), Japan (12), } \\
\text { Netherlands (10) }\end{array}$ & 5711 & $\begin{array}{l}\text { Netherlands (15), UK } \\
\text { (13), Luxembourg (9) }\end{array}$ \\
\hline
\end{tabular}

Source: Created using information from CIA (2019) and UNCTAD (2019). Figures rounded from the original.

Note: n.a. not applicable/available. Latin American countries are independent countries that were former Spanish, Portuguese, and French colonies.

Second, politically, studies of Latin American multinationals can enable new insights on the internationalization of stateowned firms and the role of government in their globalization. ${ }^{3}$ Latin American governments have a wide range of influence on the economy, from the hands-off approach in Chile to interventionism in Brazil to full control in Cuba. There are important state-owned firms in all these countries, and some of them have become significant international players, such as Chilean miner Codelco or Brazilian oil producer Petrobras. Given that much literature on the topic still examines firms from one home country, studying state-owned multilatinas can help better understand the influence of diversity in government attitudes toward state-owned firms and their global strategies.

Third, economically, the diversity in development and size of Latin American economics can yield novel comprehension of the impact of the economy on firm competitiveness. Firms that emerge in sizable countries gain an international advantage from achieving minimum efficient scale in their home market. Analyzing multilatinas can help refine such argument. For example, among the largest 500 firms in Latin America listed by the magazine AméricaEconomia ${ }^{4} 192$ are from Brazil and 122 from Mexico as expected since these are the largest Latin American countries. However, there are 69 firms from Chile, 41 from Argentina, 31 from Colombia, and 28 from Peru. A similar surprise emerges when analyzing the multilatinas among the 500 largest firms. There are 40 from Mexico and 32 from Brazil, followed by 14 from Chile, 9 from Colombia, 6 from Argentina, and 5 from Peru. This ordering is unusual given that Chile is a much smaller country, prompting a rethinking of the effect of home country size on competitiveness and internationalization.

Fourth, socially, diversity in human development across countries can help better understand the role of the home country on the development of innovations among multilatinas. Interest in understanding strategies for the base of the (economic) pyramid emerged from the experience of firms in India, which served extremely poor customers. Instead of a large base of the pyramid, Latin American countries have a significant and growing middle of the pyramid. These new middle classes enjoy disposable income and seek aspirational products with better quality and features but still at low prices. Multilatinas have responded by innovating their offerings to meet such needs, such as providing medical services and generic drugs together like Mexican pharmacy chain Farmacias Similares. Such experiences help conceptualize the drivers of innovations for poor customers.

\section{Conclusions}

Multilatinas are a newish set of global competitors that have received relatively little attention in comparison to multinationals from other emerging countries. This is unfortunate not only because these firms are increasingly becoming global challengers to established firms and deserve better attention, but also because their study can help reveal new insights into the impact of the home country on global strategy in ways that are unavailable from studying firms from other regions. The commonalities among countries in Latin America help compare the experiences of firms from multiple countries, while the variation in particular home country dimensions facilitates identifying the influence of such dimensions on firms' internationalization. Managers of multilatinas can derive insights that are more useful by learning from the experiences of similar countries and avoid imitating strategies of firms in very different emerging markets, no matter how successful those firms have become and how much the press and consultants promote them. Academics can play a leading role in providing relevant insights by developing educational materials that reflect the realities of the region, rather than assuming that lessons from firms in advanced economies or the largest emerging economies have universal applicability.

continued on page 17 